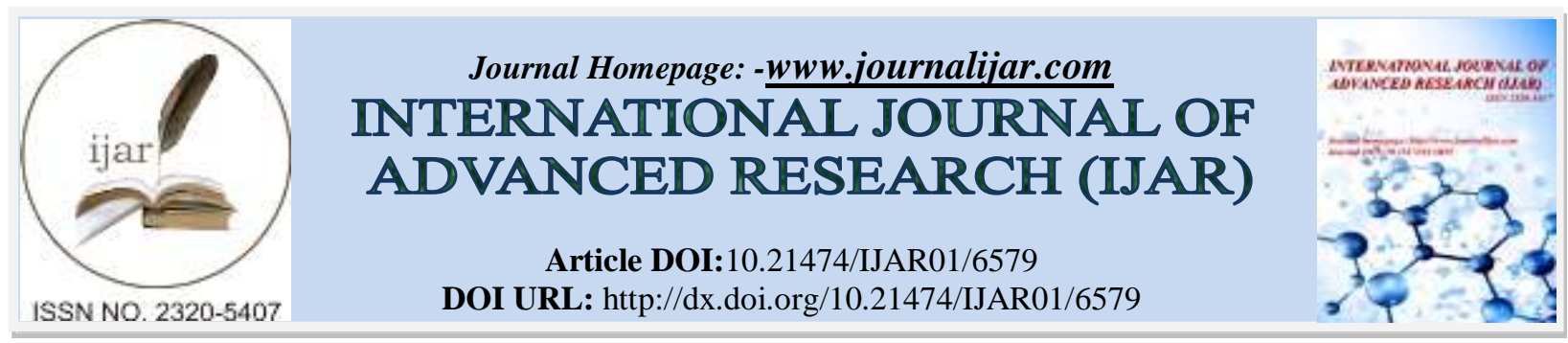

RESEARCH ARTICLE

\title{
CASE REPORT OF: CLEIDOCRANIAL DYSPLASIA PRESENT WITH HIP COXA VARA.
}

\author{
Dr. Bandar A Al-zahrani1, Dr. Abdullah Al-Babtain1, Dr. Abdullah Al-Binsaleh2
}

\section{Manuscript Info}

\section{Manuscript History}

Received: 18 December 2017

Final Accepted: 20 January 2018

Published: February 2018

Key words:-

Cleidocranial dysplasia, coxavara, hip (intertrochanteric) valgus osteotomy

\begin{abstract}
Antibiotic sensitivity revealed that allisolates were fully susceptible to enerofloxacin, ciprofloxacin, while all isolates were fully resistant to penicillin. E. coliserogrouped isolates were subjected to PCR for detection of Stx 1 and Stx 2 genes. 3 out of 7 serogrouped isolates (42.85\%) were carried Stx2 gene (O55 and O27 from contact human and 086 from mastitic milk) while Stx1 gene was not detected. phylogenetic analysis for the sequence data of the $S x t 2$ gene of $E$. coliserogroupes revealed that $S x t 2$ gene isolated from mastitic milk of cattle is closely identical (100\% identity) to $S x t 2$ gene isolated from contact human. In Conclusion, isolation of STEC from cattle might have potential pathogenicity for human. So that contact human should use sound hygienic measures during milking and management of these animals to avoid zoonotic infection.
\end{abstract}

Copy Right, IJAR, 2018,. All rights reserved.

\section{Introduction:-}

Cleidocranial dysplasia (CCD, OMIM 119600) is a rare skeletal disorder of autosomal dominant inheritance with an estimated incidence of 1/1,000,000. The main clinical features of CCD include persistent, open cranial sutures with bulging calvaria; hypoplasia or aplasia of the clavicles; dental anomalies; a short middle phalanx in the fifth finger; and associated vertebral anomalies.[1]. This condition is primarily caused by mutations in the gene that encodes runt-related transcription factor 2 (RUNX2, MIM \#600211), located on chromosome 6p21. The RUNX2 protein functions mainly in osteoblast differentiation and regulation, which contributes to the skeletal and dental abnormalities observed in CCD.[2]. In addition to its expression in osteoblasts, the RUNX2 protein is also present in non-osteoblast cells, including skeletal and smooth muscle cells, T cells, sperm cells, and neurons.[3].

Congenital coxavara is a developmental abnormality characterized by a decreased femoral neck-shaft angle, shortening of the femoral neck, and shortening of the affected lower limb. The exact cause of congenital coxavara is unknown but is believed to result from a primary defect in enchondral ossification of the medial part of the femoral neck.[4]. Histologic studies have shown that there are abnormalities in both cartilage production and metaphyseal bone formation at the proximal femur. Weight bearing on the affected hip causes increased shearing forces through the physis and leads to the progressive varus deformity.[5,6]. There are many other proposed etiologies, including metabolic, mechanical, and developmental abnormalities.

Congenital coxavara is a relatively rare deformity, occurring in approximately 1 in 25,000 live births (approximately 20 times less common than developmental hip dysplasia). Patients with congenital coxavara often have gait abnormalities or an LLD. The condition is usually diagnosed between the ages of 2 and 8 years. There is a slight increased incidence of congenital coxavara in African Americans and a positive family history. There is bilateral 
involvement in approximately $30-50 \%$ of patients. Here, we describe Cleidocranial dysplasia with left coxavara.[7,8] We reports a case of a A 17-year-old boy is a known case of cleidocranial dysplasia was complaining of limbing since childhood, progressive, and associated with left hip pain if he sits crossed leg for long time. the diagnosis of CCD was established by clinical, radiological and molecular elements, in this clinical case. He underwent a left hip (intertrochanteric) valgus osteotomy.

\section{Case presentation:-}

A 15-year-old boy presented with left hip pain. The patient has skeletal anomalies. Past history was full-term, spontaneous vaginal delivery, pregnancy unremarkable, birth weight about $3 \mathrm{~kg}$ and had normal motor development milestone. By the age of one year parents noticed delayed closure of anterior fontanelle and subsequently were found to have leg length discrepancy (LLD) and absent clavicles. On examination, he was having dysmorphic features (macrocephaly, wide metopic suture, frontal bossing head, hypertelorism, depressed nasal bridge, pulled upper lip and bilateral absent clavicles) with short limb. X-ray showed severe coxavara of the left hip (figure 1). Then, the patient was referred for gene study and he was found to be positive RUNX2 (the gene responsible for cleidocranial dysplasia).

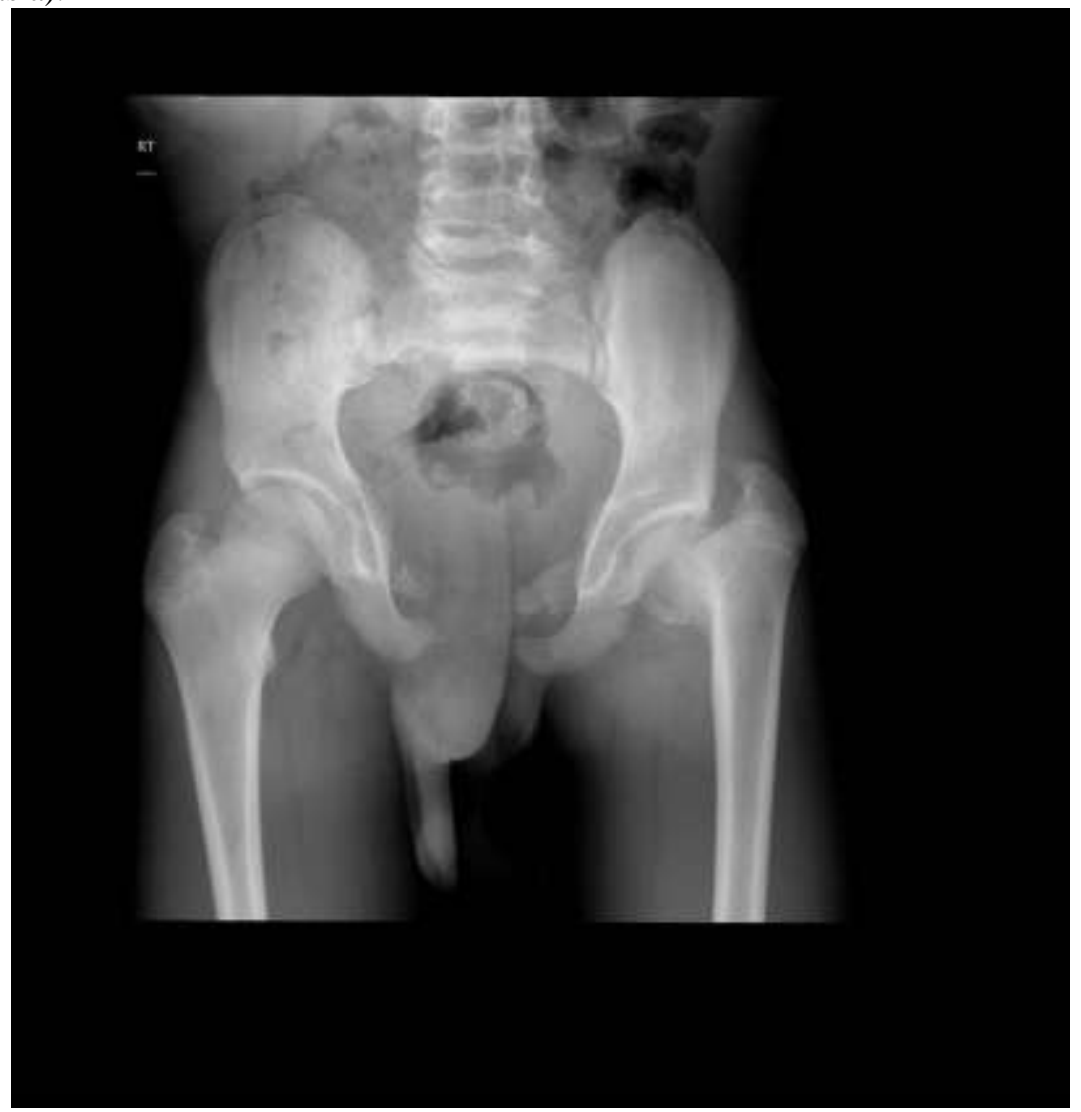

Figure 1:- a radiograph of the patient's pelvic showing severe coxavara of the left hip with a femoral neck-shaft angle of $90^{\circ}$, and a Hilgenreiner epiphyseal angle greater than $15^{\circ}$

After the patient finished all his investigations, he came back to the clinic. At that time, he started to complain of left hip pain if he sits crossed leg for long time, with leg length discrepancy $2 \mathrm{~cm}$, limping and difficulty in sitting. On examination, he was found to have limited abduction. The x-ray showed left hip coxavara $90^{\circ}$. Then, patient was booked for valgus osteotomy at proximal femur. He underwent subtrochanteric valgus osteotomy (using pediatric hip plate - figure 2). After the surgery, He was recovering and doing well. Then, he was referred to the physical therapy department for NWB mobilization to the left lower limb. 


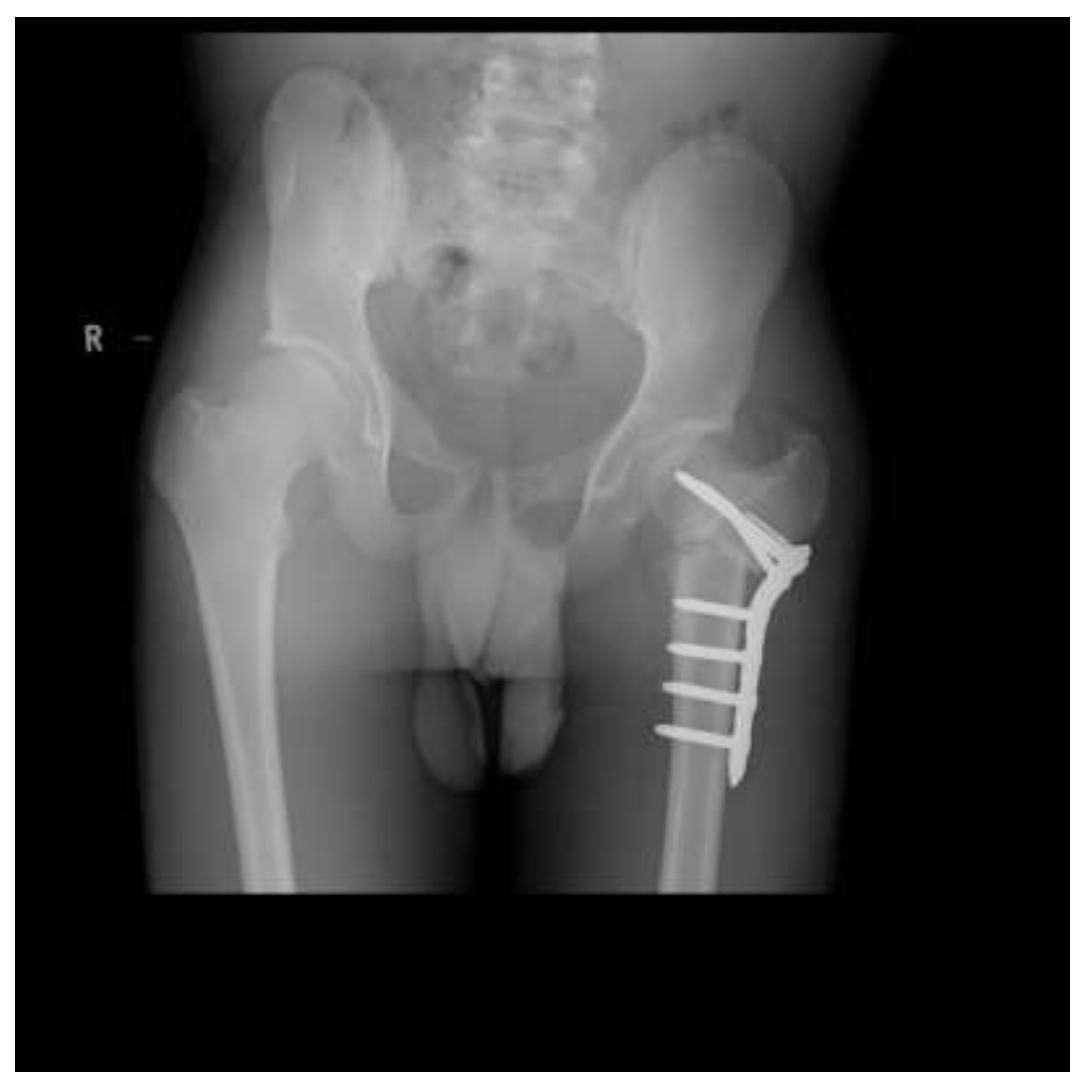

Figure 2:- a radiograph of the patient's pelvic showing the left hip after the subtrochanteric valgus osteotomy.

Before the surgery the patient was complaining of hip pain, limping, dificulity in sitting and abnormal gait. During the surgery, the femoral neck-shaft angle (FNSA) was corrected, the $2 \mathrm{~cm}$ LLD was corrected, so the Left lower limb is longer than before. After the surgery, the pain was relieved, the gait was corrected, the Limping, and difficulty in sitting were improved. No recurrence of the deformity was observed during the follow-up appointment at the outpatients department. All these Are signs of a successful surgery.

\section{Discussion:-}

Cleidocranial dysplasia (CCD) is a rare inherited disorder. The main clinical features of CCD include persistent, open cranial sutures with bulging calvaria; hypoplasia or aplasia of the clavicles; dental anomalies; a short middle phalanx in the fifth finger; and associated vertebral anomalies. Congenital coxavara is a relatively rare developmental abnormality characterized by a decreased femoral neck-shaft angle, shortening of the femoral neck, and shortening of the affected lower limb.[9]. This condition is primarily caused by mutations in the gene that encodes runt-related transcription factor 2 (RUNX2, MIM \#600211), located on chromosome 6p21. The RUNX2 protein functions mainly in osteoblast differentiation and regulation, which contributes to the skeletal and dental abnormalities observed in CCD.[10]. Histologic studies have shown that there are abnormalities in both cartilage production and metaphyseal bone formation at the proximal femur. Weight bearing on the affected hip causes increased shearing forces through the physis and leads to the progressive varus deformity.[5,6]. There are many other proposed etiologies, including metabolic, mechanical, and developmental abnormalities. There are many diagnostic criteria for this codition. Manifested by certain abnormalities such as: $94 \%$ have supernumerary teeth and eruption failure of permanent teeth; Clavicular hypoplasia are usually encountered In CCD patients which manifested as narrow shoulders on examination[11]. CCD patients are more likely to have other orthopedic deformities, such as clubfoot, genu valgus, sveer scoliosis and coxavara as presented in this case.

The few published literatures regarding developmental coxavara discussed the treatment of femoral varus deformity without addressing the acetabular side of the joint. Regarding the manegment ofcoxavara, not all cases require surgical correction; there are reported cases of spontaneous improvement of mild angulatory deformities.[12]. Amstutz and Wilson highly recommend surgery for neck-shaft angles less than $90^{\circ}$.[13]. 
In a more recent literature, Weinstein et al described an angle named the Hilgenreiner-epiphyseal (HE) angle, which is located between Hilgenreiner's line and the proximal femoral physis. Although they failed to differentiate cases of congenital coxavara with short femur from those of the developmental codition, their surgical recomendation were similar to those of Amstutz and Wilson.[14]

Management of CCD patients requires multidisciplinary approach, including the patient and the patient's family,[15] with the ministry of treatment aimed towered improving quality of life of CCD patiants . Presently, orthodontic and orthopaedic management is being undertaken for this patient.

\title{
Conclusion:-
}

cleidocranial dysplasia is a genetic condition characterized by multiple skeletal and dental anomalies. Although not as common as the dental aspects of the condition which require intervention, orthopaedic surgeons should have a working knowledge of the cleidocranial dysplasia and follow patients carefully as they grow for development of brachial plexopathy, abnormal gait or hip range of motion, signs of "chef's hat" femoral head deformity, coxavara, or osteoporosis. Patients with coxavara should be considered for surgery, whereas patients with clavicular hypoplasia and absence of pubic symphysis ossification can usually be observed and managed conservatively.

\section{References:-}

1. Brueton LA, Reeve A, Ellis R, Husband P, Thompson EM, Kingston HM. Apparent cleidocranial dysplasia associated with abnormalities of 8q22 in three individuals. Am J Med Genet. 1992;43:612-8.

2. Mundlos S. Cleidocranial dysplasia: clinical and molecular genetics. J Med Genet. 1999;36:177-82.

3. Lee B, Thirunavukkarasu K, Zhou L, Pastore L, Baldini A, Hecht J, et al. Missense mutations abolishing DNA binding of the osteoblast-specific transcription factor OSF2/CBFA1 in cleidocranial dysplasia. Nat Genet. 1997;16:307-10.

4. Komori T. Regulation of osteoblast differentiation by transcription factors. J Cell Biochem. 2006;99:1233-9.

5. Tanaka T, Sato H, Doi H, Yoshida CA, Shimizu T, Matsui H, et al. Runx2 represses myocardin-mediated differentiation and facilitates osteogenic conversion of vascular smooth muscle cells. Mol Cell Biol. 2008;28:1147-60.

6. Satake M, Nomura S, Yamaguchi-Iwai Y, Takahama Y, Hashimoto Y, Niki M, et al. Expression of the Runt domain-encoding PEBP2 alpha genes in T cells during thymic development. Mol Cell Biol. 1995;15:1662-70.

7. Jeong JH, Jin JS, Kim HN, Kang SM, Liu JC, Lengner CJ, et al. Expression of Runx2 transcription factor in non-skeletal tissues, sperm and brain. J Cell Physiol. 2008;217:511-7.

8. Herring, J. A. (2002). Congenital coxavara. In Tachdjian's pediatric orthopaedics (3rd Ed.). Philadelphia: WB Saunders Company; 265-275.

9. Staheli, L. T. (2001). Practice of pediatric orthopaedics. Philadelphia: Lippincott Williams \& Wilkins.10. Beals, R. K. (1998). Coxavara in childhood: Evaluation and management. Journal of the American Academy of Orthopaedic Surgeons, 6(2), 93-99

10. Ortega RI, Obando FS. Displasiacleidocraneal: presentación de uncaso. UniversitasMédica. 2016; 57: 115-22.

11. Bufalino A, Paranaíba LM, Gouvêa AF, et al. Cleidocranial dysplasia: oral features and genetic analysis of 11 patients. Oral Dis 2012;18:184-90.

12. LeMesurier AB: Developmental coxavara. J Bone Joint Surg 1948; 30B:595-605.

13. Amstutz HC. Freiberger RH: Coxavara in children. ClinOrthop 1966: 22:73-92.

14. Weinstein JN. Kuo KN. Millar EA: Congenital coxavara. A retrospective review. J PediatrOrthop 1984; 4:7077.

15. Gömleksiz C, Arslan E, Arslan S, et al. Delayed diagnosis of cleidocranial dysplasia in an adult: A case report. ActaMedicaAcademica 2014;43:92-6.

\author{
Abbreviations \\ CCD: Cleidocranialdysplasia . \\ RUNX2: Runt-related transcription factor 2 \\ FNSA: the femoral neck-shaft angle. \\ LLD: leg length discrepancy
}

\section{Ethical Responsibilities}

The authors state that they have 
followed the protocols of their Center and Local regulations

on the publication of patient data. The

authors have obtained the informed consent of the

patients and/or subjects referred to in the article

\section{Financial Disclosure}

Null

\section{Conflicts of Interest}

The authors declare that they have no conflict of interest with respect to this case report.

\section{Acknowledgements}

To the Faculty of Genatic department King Fahad Specialist Hospital - Dammam, Saudi Arabia

\section{Competing interests}

The authors declare that they have no competing interests.

\section{Authors' contributions}

BA: following up the patient and was a major contributor in writing the manuscript.

AAT: conducted the examination and did the biopsy and writhe the case history section.

AAS: the primary clinician who encounter the patient and write the description of the imaging in the manuscript. 